Pavia, Fondazione IRCCS Policlinico San Matteo, Obstetrics and Gynaecology, Pavia, Italy

Background: Systemic Sclerosis (SSc) is one of the rheumatic diseases burdened with obstetrical complications. An Italian multicenter study showed that women with SSc have a higher-than-normal risk of intrauterine growth restriction, preterm delivery, very-low birth weight babies and pregnancy should be discouraged in patients with severe organ damage. However, with a multidisciplinary management, patients with SSc can have successful outcomes ${ }^{1}$. Little is known about the pathogenesis of obstetrical complications, as studies on placenta are case reports or description of a few cases $^{2,3}$.

Objectives: The aim of this study was to analyze the placental alterations with a focus on the role of inflammation in the pathogenesis of obstetrical complications in SSc, including the study of the atypical chemokine receptor 2 (ACKR2), involved in immune modulation and known to be highly expressed in circulating leucocytes in SSc patients ${ }^{4-6}$

Methods: Eight SSc pregnant patients were compared with 16 patients with other rheumatic diseases (ORD) and 16 healthy controls $(\mathrm{HC})$, matched for gestational age. Clinical data were collected. Placentas biopsies were obtained for histopathological analysis and immunohistochemistry for CD3, CD20, CD11c, CD68 and ACKR2. Frozen placenta samples from 4 SSc, 8 ORD and $8 \mathrm{HC}$ were analyzed by qPCR for ACKR2 gene expression and proteins were extracted for multiplex assay for cytokines, chemokines and growth factors involved in angiogenesis and inflammation. Statistical analysis was performed with parametric or non-parametric tests depending on samples distribution.

Results: The number of placental CD3 ( $p<0.05)$, CD68 $(p<0.001)$ and CD11c+ $(p<0.001)$ cells was significantly higher considering the group of patients affected by rheumatic diseases (SSc+ORD) compared to HC. The SSc group alone did not show significance due to the lower sample size. No differences were observed between groups in terms of vascular alterations or fibrosis. The percentage of stained area for ACKR2 and the ACKR2 transcripts levels were comparable between groups. Hepatocyte growth factor (HGF), involved in angiogenesis, was significantly increased in the group of rheumatic diseases patients $(S S c+O R D)$ compared to $H C(p<0.05)$, while the chemokine CCL5 was significantly higher in SSc patients compared to patients affected by ORD $(p<0.05)$ and to HC $(p<0.01)$. CCL5 levels directly correlated with the number of all inflammatory cells considered and higher levels were associated to histological villitis $(p<0.01)$

Conclusion: The higher number of placental inflammatory cells and the alterations in the levels of HGF and especially CCL5 could play a role in the pathogenesis of the obstetrical complications in SSc. ACKR2 does not seem involved in the obstetrical complications of SSc.

References:

[1] Taraborelli M, et al. Arthritis Rheum. 2012

[2] Ibba-Manneschi L, et al. Ann Rheum Dis. 2010

[3] Doss BJ, et al. Hum Pathol. 1998

[4] Graham GJ. Eur J Immunol. 2009

[5] Martinez de la Torre Y, et al. Proc Natl Acad Sci U S A. 2007

[6] Codullo V, et al. Ann Rheum Dis. 2011

Disclosure of Interests: None declared

DOI: 10.1136/annrheumdis-2020-eular.4379

\section{SAT0281 BIOSAMPLES FROM AT RISK SSC PATIENTS SHOW CLASSIC PATHOLOGICAL SIGNS OF SCLERODERMA: OPPORTUNITY FOR DIAGNOSIS OF PRE-CLINICAL SSC}

R. Ross ${ }^{1}$, I. Georgiou ${ }^{1}$, A. Carriero ${ }^{1,2}$, G. Abignano ${ }^{1}$, C. Wasson ${ }^{1}$, G. Migneco ${ }^{1}$, A. Herrick ${ }^{3}$, C. Denton ${ }^{4}$, F. Del Galdo ${ }^{1,5} .{ }^{1}$ University of Leeds, Leeds Institute of Rheumatic and Musculoskeletal Medicine and Biomedical Research Centre, Leeds, United Kingdom; ${ }^{2}$ San Carlo Hospital of Potenza, Rheumatology Institute of Lucania (IReL) and Rheumatology Department of Lucania, Lucania, Italy; ${ }^{3}$ The University of Manchester, Division of Musculoskeletal \& Dermatological Sciences (L5) Division of Musculoskeletal \& Dermatological Sciences, Manchester, United Kingdom; ${ }^{4}$ University College London, Experimental Rheumatology, UCL Division of Medicine, London, United Kingdom; ${ }^{5}$ Chapel Allerton Hospital, NIHR Leeds Biomedical Research Centre, Leeds, United Kingdom

Background: The VEDOSS study has recently indicated that more than $80 \%$ of patients affected by Raynaud's phenomenon (RP) with specific SSc auto-antibodies and capillaroscopy changes satisfied ACR/EULAR 2013 criteria within 5 years. These data suggest that there is a window of opportunity for early detection of SSc in these patients.

Objectives: Here we aimed to determine whether sera, skin biopsies and skin fibroblasts cultured from these patients showed any biomarker sign of SSc.
Methods: Fifty-nine at risk patients identified by having RP and SSc auto-antibodies or capillaroscopy pattern (or both) were enrolled in the Kennedy national inception cohort. Sera were tested for IFN inducible chemokines (CXCL-9,10 and 11 and CCL2, 8 and 19) and biomarker of extracellular matrix turnover (ELF test), all previously shown to be increased in SSc. Further, two $3 \mathrm{~mm}$ skin biopsies were taken from the forearms from $3 \mathrm{ACA}+\mathrm{ve}$ (anti-centromere antibodies), 3 SCL70+ve patients. One biopsy was subjected to histology analysis, including haematoxylin and eosin staining and immunohistological staining for Collagen Type 1, alpha-SMA, Caveolin 1 and CD31 as endothelial marker. The other biopsy was used to explant fibroblasts cultures. mRNA and protein were isolated from primary fibroblasts and processed for RT-qPCR and western blotting analyses.

Results: Sera from at risk patients showed overall higher IFN inducible chemokines and ELF test $(P<0.05)$ with bimodal distribution among patients. Skin biopsies from both ACA or SCL70+ve patients showed decreased number of CD31+ cells, increased number of myofibroblasts and increased colla gen bundles within the dermis, as usually seen in SSc, compared to healthy controls. In vitro, fibroblasts from both ACA or SCL70+ve patients showed average 10-fold higher collagen mRNA levels and 31-fold increased collagen protein levels

compared to healthy control fibroblasts. Furthermore, fibroblasts from ACA or SCL70+ve patients showed limited TGF-beta induced increase in collagen and SMA expression, similar to SSc fibroblasts.

Conclusion: Although pilot in nature, this study suggests that patients "at risk" already show biomarker signs of SSc both in their sera, at skin biopsy and fibroblast level. Longitudinal studies on patients at this stage of pre-clinica disease may inform on the stratification strategies for imminent progression to clinical manifestations, and offer both insights on pathogenesis of clinica signs and a window of opportunity for delaying the onset clinical intervention trials.

Disclosure of Interests: rebecca ross: None declared, loanna Georgiou: None declared, Antonio Carriero: None declared, Giuseppina Abignano: None declared, Chris Wasson: None declared, Gemma Migneco: None declared, Ariane Herrick: None declared, Christopher Denton Grant/research support from: GlaxoSmithKline, CSL Behring, and Inventiva, Consultant of: Medscape, Roche-Genentech, Actelion, GlaxoSmithKline, Sanofi Aventis, Inventiva, CSL Behring, Boehringe Ingelheim, Corbus Pharmaceuticals, Acceleron, Curzion and Bayer, Francesco Del Galdo: None declared

DOI: 10.1136/annrheumdis-2020-eular.5666

\section{SAT0282 \\ ASSOCIATION BETWEEN A VARIANT OF THE SRP55 SPLICING FACTOR GENE AND SYSTEMIC SCLEROSIS IN AN ITALIAN POPULATION}

E. Romano ${ }^{1}$, M. Manetti ${ }^{1}$, J. Kosalka-Wegiel ${ }^{2}$, B. S. Fioretto ${ }^{1}$, I. Rosa ${ }^{1}$,

E. Sticchi ${ }^{1}$, S. Guiducci ${ }^{1}$, S. Bellando-Randone ${ }^{1}$, L. Ibba-Manneschi ${ }^{1}$,

M. Matucci-Cerinic ${ }^{1} .{ }^{1}$ University of Florence, Department of Experimental and

Clinical Medicine, Florence, Italy; ${ }^{2}$ Jagiellonian University Medical College,

Department of Medicine, Cracow, Poland

Background: In systemic sclerosis (SSc), alternative splicing of the last exon (exon 8) of vascular endothelial growth factor (VEGF)-A pre-mRNA is a key element in the switch from proangiogenic to antiangiogenic VEGF-A isoforms. The mRNA-binding protein serine/arginine protein 55 (SRp55, also known as SFRS6) is a key regulatory splicing factor that promotes distal splice-site selection in the exon 8 region of VEGF-A pre-mRNA and subsequent upreg ulation of the exon 8b-containing $V_{E G F}{ }_{165} b$ antiangiogenic isoform. Overexpression of both VEGF ${ }_{165} \mathrm{~b}$ and SRp55 has been implicated in SSc-related angiogenesis impairment and peripheral vascular damage. Moreover, differential splicing of the VEGF-A gene has been shown to be critical for development of pulmonary fibrosis. Of note, previous studies reported the lack of sequence variations in the VEGF-A alternatively spliced region, while a single nucleotide polymorphism (SNP) in the SRp55 gene (rs2235611) has been associated with susceptibility to disturbed ocular angiogenesis in proliferative diabetic retinopathy.

Objectives: This case-control pilot study examined the possible implication of SRp55 rs2235611 SNP in the genetic predisposition to SSc susceptibility and clinical phenotype.

Methods: A total population of 872 white Italian individuals (414 SSc patients, 458 controls) was studied. All patients were classified as limited and diffuse cutaneous SSc (IcSSc and dcSSc, respectively) and were clinically evaluated for the presence of autoantibodies (anticentromere, anti-Scl70 antibodies), pulmonary fibrosis and digital ulcers. The SRp55 rs2235611 SNP was genotyped by TaqMan Real-Time PCR.

Results: SRp55 rs2235611 genotype distribution and allele frequency were similar in SSc and healthy controls, though a trend toward significance was observed for genotype distribution ( $p=0.07$ ). The SRp55 rs2235611 AA genotype significantly influenced the predisposition to SSc (OR 2.55, 95\% Cl 1.11 to 5.57 , 
$\mathrm{p}=0.03$ ), and to both IcSSc (OR $2.80,95 \% \mathrm{Cl} 1.16$ to $6.84, \mathrm{p}=0.02)$ and dcSSc (OR $3.42,95 \% \mathrm{Cl} 1.20$ to $9.72, \mathrm{p}=0.02$ ) subtypes. The $S R p 55$ rs2235611 A minor allele and AA genotype showed a significant risk association with susceptibility to SSc-related pulmonary fibrosis (A allele: OR $1.39,95 \% \mathrm{Cl} 1.00$ to $1.93, p=0.046$; AA genotype: $\mathrm{OR} 3.95,95 \% \mathrm{Cl} 1.48$ to $10.54, \mathrm{p}=0.006)$. A trend toward an association between the AA genotype and anti-Scl70 antibody-positive SSc was also found (OR 2.82, $95 \% \mathrm{Cl} 0.95$ to $8.37, \mathrm{p}=0.06$ ). Both rs2235611 A allele and $\mathrm{AA}$ genotype were significantly associated with the SSc subset without digital ulcers (A allele: OR 1.33, 95\% Cl 1.01 to $1.75, p=0.04$; AA genotype: OR $3.26,95 \% \mathrm{Cl}$ 1.32 to $8.03, p=0.01$ ).

Conclusion: The SRp55 rs2235611 polymorphism is associated with susceptibility to SSc and, in particular, with SSc-related pulmonary fibrosis and peripheral vascular phenotype, consistent with a role of VEGF-A pre-mRNA alternative splicing in the development of pulmonary fibrosis and impairment of angiogenesis. Further replication studies are warranted to confirm our findings in independent SSc cohorts.

Disclosure of Interests: Eloisa Romano: None declared, Mirko Manetti: None declared, Joanna Kosalka-Wegiel: None declared, Bianca Saveria Fioretto: None declared, Irene Rosa: None declared, Elena Sticchi: None declared, Serena Guiducci: None declared, Silvia Bellando-Randone: None declared, Lidia Ibba-Manneschi: None declared, Marco Matucci-Cerinic Grant/research support from: Actelion, MSD, Bristol-Myers Squibb, Speakers bureau: Acetelion, Lilly, Boehringer Ingelheim

DOI: 10.1136/annrheumdis-2020-eular.1707

\section{SAT0283 SOLUBLE GUANYLATE CYCLASE REDUCED THE GASTROINTESTINAL FIBROSIS IN BLEOMYCIN- INDUCED MOUSE MODEL OF SYSTEMIC SCLEROSIS}

Y. Yamamoto ${ }^{1}$, T. Okano ${ }^{1}$, T. Nagamoto ${ }^{1}$, Y. Fujikawa ${ }^{1}$, Y. Ichise ${ }^{1}$, H. Yamada ${ }^{1}$, I. Naka ${ }^{1}$, Y. Ueda' ${ }^{1}$ K. Akashi', S. Sendo ${ }^{1}$, A. Onishi ${ }^{1}$, J. Saegusa', A. Morinobu ${ }^{1}$. ${ }^{1}$ Department of Rheumatology and Clinical Immunology, Kobe University Graduate School of Medicine, Kobe, Japan

Background: Systemic scleroderma (SSc) is a chronic autoimmune-mediated connective tissue disorder. Although the etiology of the disease remains undermined, SSc is characterized by fibrosis and proliferative vascular lesions of the skin and internal organs. SSc involves the gastrointestinal tract in more than $90 \%$ of patients ${ }^{1}$. Soluble guanylate cyclase (sGC) is used to treat pulmonary artery hypertension (PAH), and has been shown to inhibit experimental skin fibrosis ${ }^{2}$.

Objectives: The aim of this study is to investigate whether bleomycin (BLM)treated mice show gastrointestinal fibrosis, and find a therapeutic strategy to the lesion.

Methods: Female C57BL/6J mice were treated with BLM or normal saline by subcutaneous implantation of osmotic minipump. These mice were sacrificed on day 28 or day 42 . Gastrointestinal pathologies were examined by Masson Trichrome staining. The expression of fibrosis-related genes in gastrointestinal tract were analyzed by real-time PCR, and the levels of collagen in the tissue was measured by Sircol collagen assay. To evaluate peristaltic movement, the small intestinal transport (ITR\%) was calculated as [Dyeing distancex(Duodenum- Appendix)] $-1 \times 100(\%)$. We treated BLM-treated mice with soluble guanylate cyclase (sGC) or DMSO orally and analyzed them on day 42.

Results: Histological examination revealed that fibrosis from lamina propria to muscularis mucosa in the esophagus was significantly increased in BLMtreated mice, suggesting that BLM induces esophageal fibrosis in C57BL/6J mice. In addition, the levels of Col3a1 and CTGF were significantly increased in BLM-treated mice. More severe fibrosis was observed in the mice sacrificed on day 42 than the mice sacrificed on day 28 . The ITR\% was found to be significantly lower in BLM-treated mice, suggesting that gastrointestinal peristaltic movement was reduced in BLM-treated mice. Furthermore, we demonstrated that SGC treatment significantly reduced fibrosis of esophagus and intestine in BLM-treated mice, by histological examination and Sircol collagen assay.

Esophagus (Masson's trichrome stain $\times 100$ )
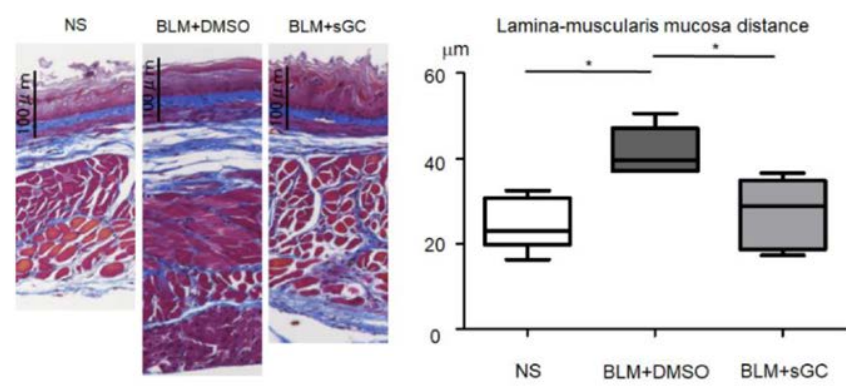

* One way ANOVA Newman-Keuls

Conclusion: These findings suggest that BLM induces gastrointestinal fibrosis in C57BL/6J mice, and treatment with SGC improves the BLM-induced gastrointestinal lesion.

\section{References:}

[1] Anton Emmanuel. Current management of the gastrointestinal complications of systemic sclerosis. Nat Rev Gastroenterol Hepatol. 2016; 13: 461-472.

[2] Clara Dees, et al. Stimulators of Soluble Guanylate Cyclase (sGC) Inhibit experimental Skin Fibrosis of Different Aetiologies. Ann Rheum Dis. 2015;74 (8): 1621-5

Acknowledgments: : None.

Disclosure of Interests: None declared

DOI: 10.1136/annrheumdis-2020-eular.2768

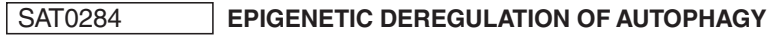 PROMOTES FIBROSIS IN SYSTEMIC SCLEROSIS}

A. Zehender ${ }^{1}$, N. Y. Lin ${ }^{1}$, Y. N. LI ${ }^{1}$, A. H. Györfi ${ }^{1}$, C. Bergmann ${ }^{1}$, A. Ramming ${ }^{1}$,

G. Schett2, J. Distler2. ' University Erlangen-Nürnberg (FAU) and University Hospital Erlangen, Department of Internal Medicine 3 - Rheumatology and Immunology, Erlangen, Germany; ${ }^{1}$ University Erlangen-Nürnberg (FAU) and University Hospital Erlangen, Department of Internal Medicine 3 Rheumatology and Immunology, Erlangen, Germany

Background: Autophagy is catabolic process allowing cells to degrade unnecessary or dysfunctional cellular organelles. Failure of appropriate regulation of autophagy, however, can severely perturb tissue homeostasis. Several stimuli present in fibrosis such as pro-fibrotic cytokines are known to activate autophagy. Objectives: The objective of this work was to characterize the regulation of autophagy in systemic sclerosis (SSc) and to decipher its role in the pathogenesis of SSc.

Methods: Activation of autophagy in SSc skin and matched tissue samples from healthy individuals was assessed by immunofluorescence staining for ATG7, BECLIN1 and P62. We generated $A \operatorname{tg} 7^{\mathrm{fl} / \mathrm{fl}} \times \mathrm{Col}$ 1 a2; CreER mice to selectively disable autophagy in fibroblasts. The role of the autophagy was investigated in the model of bleomycin- and T $\beta$ Rlact-induced dermal and pulmonary fibrosis. Overexpression of Myst1 was achieved by adenovirus encoding for Myst1. Collagen release and protein expression were measure by Western blot. Target genes were analyzed by RT-PCR. Co-immunoprecipitation and reporter assay were performed to study physical and functional interactions between MYST1 and SMAD3. To monitor the autophagic flux in vitro and in vivo we generated adenoviral vectors encoding for tandem fluorescent-tagged LC3 (mRFP-EGFP-LC3).

Results: Transforming growth factor- $\beta$ (TGF $\beta$ ) activates autophagy by an epigenetic mechanism to amplify its profibrotic effects. TGF $\beta$ induces autophagy in fibrotic diseases by SMAD3-dependent downregulation of the H4K16 histone acetyltransferase MYST1, which regulates the expression of core components of the autophagy machinery such as ATG7 and BECLIN1. Activation of autophagy in fibroblasts promotes collagen release and is both, sufficient and required, to induce tissue fibrosis. Forced expression of MYST1 abrogates the stimulatory effects of TGF $\beta$ on autophagy and re-establishes the epigenetic control of autophagy in fibrotic conditions. Interference with the aberrant activation of autophagy inhibits TGF $\beta$-induced fibroblast activation and ameliorates experimental dermal and pulmonary fibrosis. These findings link uncontrolled TGF $\beta$ signaling to aberrant autophagy, deregulated epigenetics in fibrotic diseases and may open new avenues for therapeutic intervention in fibrotic diseases.

Conclusion: We demonstrate that the epigenetic control of autophagy is disturbed by a TGF $\beta$-dependent downregulation of the H4K16 histone acetyltransferase MYST1. The increased activation of autophagy induces fibroblast-to-myofibroblast transition and promotes fibrotic tissue remodeling Re-expression of MYST1 prevents aberrant autophagy, limits the profibrotic effects of TGF $\beta$ and ameliorates experimental fibrosis. Restoration of the 